\title{
PARA UMA ONTOLOGIA DOS ESTUDOS DE TRADUÇÃO ${ }^{1}$
}

\author{
THOMAS J.C. HÜSGEN
}

\section{A B S T R AC T}

The need for a re-mapping of concepts in the field of translation studies is something that becomes evident today. In this context, Sonia Vandepitte (2008) proposes a renewed thesaurus that will be analyzed, discussed and completed in this article understood as a constructive contribution to a new ontology of translation.

A necessidade de uma ontologia dos estudos de tradução justifica-se, neste momento, pela diversidade dos estudos propostos, das suas abordagens teóricas, dos seus objetos de investigação, das suas orientações metodológicas e dos contextos e práticas analisadas. Esta sua riqueza epistemológica poderá igualmente ser vista como uma debilidade que, por vezes, resulta numa relação difícil entre aqueles que se dedicam ao estudo deste domínio tão abrangente e heterogéneo, e aqueles que, mergulhados nesta diversidade, prosseguem profissionalmente uma atividade em constante mudança. Colocado perante desafios profissionais múltiplos que exigem uma adaptação dinâmica, o tradutor profissional procura respostas vindas dos estudos de tradução que nem sempre encontra. E, muitas vezes, não porque não haja trabalho que ofereça essa resposta, mas porque, perante uma explosão de projetos académicos no domínio e consequente produção científica, se tornou difícil o acesso à informação procurada.

Não é, assim, por acaso que têm vindo a ser apresentados nos últimos anos modelos de reorganização de toda a investigação disponível. Partindo da proposta mais influente de Holmes, dos anos setenta, procuraram lançar a linha para abrir o caminho neste imenso labirinto que se criou e que vai separando o mundo profissional do mundo académico.

Existem hoje em dia cerca de 20.000 publicações em revisão por pares no domínio da tradução. Contributos como os de Baker (1998), do grupo da "Bibliography of Translation Studies" da St. Jerome, Gentzler (2001), Kuhiwczak \& Littau (2007), Munday (2009), Pym (2010), Tymoczko (2005) e Williams \& Chesterman

[1] Comentando a proposta de Sonia Vandepitte "Remapping Translation Studies: Towards a Translation Studies Ontology". Comunicação proferida a 7 de fevereiro de 2014 no âmbito do Seminário (Re)Cartografar os Estudos de Tradução no Século XXI, organizado pelo Centro de Estudos de Comunicação e Cultura da Universidade Católica. 
(2002), para mencionar apenas alguns dos mais importantes, produziram assim longas listas de abordagens no âmbito dos estudos de tradução que, de acordo o artigo "Remapping Translation Studies: Towards a Translation Studies Ontology" de Vandepitte (2008), pecaram por manifesta falta de consistência:

"These overviews are very incongruent, however: the few subdivisions of types of translation studies areas that are marked clearly differ from one another, and, taken together, these contributions result in a collection of fairly long lists of translation studies approaches that lack a consistent basis."

(Vandepitte 2008, pg. 569)

Para o objetivo declarado de reorganizar algo que entrou em desordem, esta crítica é, sem dúvida, relevante e, coloca, desde já um critério de análise fundamental para a validade de uma nova ontologia como a que Vandepitte propõe.

Vandepitte baseia-se conceptualmente em Aitchison et al. (2000) para salvaguardar a concisão da sua ontologia. A construção de thesauri manuais afigura-se uma tarefa complexa resultando na construção de estruturas que implicam relações internas de vária ordem, sejam elas hierárquicas, não-hierárquicas, equivalentes e associadas. Uma das finalidades destas estruturas é a otimização da pesquisa de informação. É através da "etiquetagem" ontológica dos seus objetos que a informação se torna pesquisável e comparável contextualizando e relacionando cada item na relação que estabelece com os restantes. Desnecessário será relembrar que, para isso, se torna fundamental criar uma ordem que permita estabelecer estas relações. Identificar estas relações exige um conhecimento profundo do domínio em análise para o qual o thesaurus é construído. A usabilidade deste tipo de estruturas depende primordialmente da sua capacidade de localizar e contextualizar as suas entradas num sistema de conceitos que, pelo tipo de relação estabelecida ontologicamente, se definem mutuamente.

Depois de separar os conceitos principais dos periféricos, o domínio em análise é descrito em termos de subáreas que o definem. Segue-se o preenchimento de cada subárea por entradas que correspondem à sua definição recorrendo-se a listas existentes, manuais, resumos, catálogos, etc. Uma vez identificados todos os termos a considerar, cada um terá que ser analisado de acordo com o material restante em termos de sinónimos, hipónimos ou hiperónimos. Deste processo podem resultar imprecisões, lacunas ou mesmo erros de organização interna, sendo necessárias, por isso, sucessivas revisões que poderão conduzir a 1. acréscimo de termos, 2. identificação de mais níveis nas hierarquias já estabelecidas ou proposta de redução de níveis, 3. acréscimo/modificação de sinónimos, 4. sugestão de novas relações ou até 5 . eliminação de entradas desnecessárias. 
É neste contexto que vejo o meu contributo possível para a ontologia proposta por Vandepitte que irei organizar de acordo com os pontos 1 e 2 acima referidos, designadamente os pontos relativos a [1] acréscimo de termos, e a [2] identificação de mais níveis nas hierarquias já estabelecidas ou proposta de reduções de níveis. Dentro desta argumentação fundamental poderão surgir, casuisticamente, aspetos de modificação de sinónimos (ponto 3) ou mesmo a sugestão de novas relações associadas (ponto 4).

No entanto, antes de o fazer gostaria de esclarecer desde já que não pretendo formular uma organização alternativa, certamente possível, mas antes propor algumas alterações que, no meu entendimento, aumentam a sua operacionalidade e subsequente adequação ao objetivo, expresso pela autora, de desenvolver uma alternativa à organização proposta de Holmes (1987) identificando categorias de classificação de acordo com critérios precisos que permitirão a apresentação de todo o tipo de estudos no âmbito da tradução num novo mapa coerente e consistente (cf. Vandepitte 2008, pg. 573).

\section{[1] ACRÉSCIMO DE TERMOS}

Neste campo parece-me pertinente apontar para a falta de um termo no âmbito dos estudos organizados pelo seu propósito (types by purpose), ou seja, aqueles que tradicionalmente se enquadram nos estudos aplicados da tradução. Postulando que os estudos aplicados diferem dos estudos puros na maneira como procuram aplicar o conhecimento adquirido na formulação de normas, padrões e aplicações práticas, são considerados três campos de investigação: (i) modelos de ensino de tradução (translation teaching models), (ii) ética tradutiva (translation ethics) e (iii) tradução cultural (cultural translation). O termo tradução cultural neste contexto poderá causar alguma perplexidade dado o seu caráter abrangente e pouco definido no sentido restrito de uma norma, a não ser que nos proponhamos considerálo na perspetiva da teoria performativa em que por exemplo se enquadra a questão dos cânones tradutivos e da influência das normas culturais na aceitabilidade de textos traduzidos. Enquanto os modelos de ensino de tradução, bem como a definição ética podem ser considerados em termos de propósito, a tradução cultural pela abrangência do conceito "cultura" não permite essa restrição. Tendo por isso alguma reticência acerca da inclusão do termo neste campo, parece-me importante, antes de mais, considerar aqui o que poderíamos chamar a avaliação qualitativa de produtos tradutivos (translation quality assessment), para não usar o termo crítica da tradução, especialmente conotado com o campo específico da tradução literária. Curiosamente Vandepitte apresenta num primeiro momento o termo crítica da tradução (T[ranslation] criticism) (cf. Vandepitte 2008, pg. 574) em paralelo com o termo tradução cultural (cultural T[ranslation]), deixando-o cair neste contexto na listagem final, sem explicação aparente. 0 termo avaliação qualitativa de produtos tradutivos (translation assessment) aparece como termo asso- 
ciado à didática (studies of translation teaching), e como sendo sinónimo de avaliação tradutiva (translation evaluation), por sua vez o termo qualidade tradutiva "translation quality" surge como termo associado aos estudos da profissão de tradutor (translation profession studies), e como sinónimo de avaliação qualitativa de produtos tradutivos (translation quality assessment). o termo estudos da avaliação de qualidade tradutiva orientados para o efeito (effect-oriented translation quality assessment studies) aparece como hiperónimo da entrada estudos orientados para o resultado do processo tradutivo (result-oriented translation studies). No âmbito dos estudos multifocalizados encontramos o termo crítica de tradução (translation criticism) como termo hiperónimo da tradução literária (literary translation). E como exemplos de estudos multifocalizados no discurso e resultado são apresentados os termos associados revisão tradutiva e critica (translation reviewing and criticism) e avaliação de qualidade (quality assessment), sendo este sinónimo de avaliação tradutiva (translation evaluation). Torna-se, a meu ver, imprescindível, introduzir a avaliação de textos traduzidos no campo de investigação que se propõe alargar o conhecimento e introduzir mudança ao apresentar critérios de avaliação de textos traduzidos. No contexto da crescente utilização da tradução automática no campo profissional, estes estudos abarcarão os processos de pós-edição em que a aplicação de modelos de avaliação contribuem para processos de revisão e avaliação mais eficazes.

Em relação aos tipos por sujeito (types by subject), Vandepitte sugere a distinção entre os estudos de estratégias tradutivas (studies of translation strategies) e os estudos de técnicas linguísticas de tradução (studies of linguistic translation techniques):

NT: studies of translation strategies

RT: adaptation

RT: domestication

RT: equivalence

RT: explicitation

RT: foreignization

RT: free translation

RT: imitation

RT: literal translation

UF: word-for-word translation

UF: metaphrase

RT: paraphrase

RT: sense-for-sense translation

NT: studies of linguistic translation techniques

NT: compensation

RT: shifts of translation

(Vandepitte 2008, pg. 585) 
Esta distinção afigura-se-me discutível devido à confusão instalada na utilização dos conceitos estratégia, método, procedimento e técnica. Segundo Venuti (1998) as estratégias envolvem decisões fundamentais relacionadas com as macroestruturas textuais que o autor classifica como sendo de domesticação (domestication) ou estranhante (foreignization). Por sua vez, Jääskeläinen (1993) distingue estratégias globais que se reportam a princípios e modos de ação gerais, enquanto que as estratégias locais se referem a opções mais específicas relacionadas com a tomada de decisão no contexto da solução de problemas ao nível das microestruturas textuais: "global strategies refer to general principles and modes of action and local strategies refer to specific activities in relation to the translator's problem-solving and decision-making." Jääskeläinen (1993, pg. 16). Newmark (1988) propõe a distinção entre método tradutivo e procedimento distinguindoos de forma semelhante à de Jaaskelainen para diferenciar estratégias globais de estratégias locais: "while translation methods relate to whole texts, translation procedures are used for sentences and the smaller units of language." (Newmark 1988, pg. 81). A diferenciação entre opções macro- e micro-estruturais parece-me importante no contexto dos estudos orientados para a descrição do processo e que creio que devia ser visível na apresentação de um thesaurus deste tipo. Assim tornar-se-ia, a meu ver, necessário reordenar a lista de Vandepitte no sentido de a tornar mais consistente e clarificadora, de acordo com a seguinte proposta:

NT: studies of translation strategies

RT: adaptation

RT: domestication

RT: equivalence

RT: explicitation

RT: foreignization

RT: free translation

RT: imitation

RT: literal translation

UF: word-for-word translation

UF: metaphrase

RT: paraphrase

RT: sense-for-sense translation

NT: studies of linguistic translation techniques

NT: compensation

RT: shifts of translation
NT: studies of translation strategies

RT: adaptation

RT: domestication

RT: foreignization

RT: free translation

RT: imitation

RT: literal translation

UF: word-for-word translation

UF: metaphrase

RT: sense-for-sense translation

NT: studies of linguistic translation procedures

NT: compensation

RT: shifts of translation

(Vandepitte 2008, pg. 585)

(proposta do autor) 
Para além disso, não me parece que o procedimento da compensação (compensation) seja aquele que subsume todos os procedimentos micro-estruturais propostos pelos diversos modelos, sendo um conceito retomado por Hervey \& Higgins (1992) para explicar procedimentos que compensem a perda de características importantes do texto de partida (TP) criando efeitos semelhantes no texto de chegada $(\mathrm{TCH})$ por meios diferentes:

"Compensation" here means "the technique of making up for the translation loss of important ST [source text] features by approximating their effects in the TT [target text] through means other than those used in the ST".

(Hervey \& Higgins 1992, pg. 248)

Seria, por isso, um tipo de procedimento entre outros, como p.ex.:
RT: Transference
RT: Naturalization
RT: Cultural equivalent
RT: Functional equivalent
RT: Descriptive equivalent
RT: Componential analysis
RT: Synonymy
RT: Through-translation

para referir apenas alguns.

Para além deste aspeto, a utilização do termo alterações tradutivas (translation shifts) para definir todo o tipo de procedimentos micro-estruturais afigurase-nos no mínimo ambígua, por este termo poder ser confundido com conceitos macro-estratégicos de Catford (1965) e Popovic (1976) ou com o conceito microestratégico de mudanças ou transposições (shifts or transpositions) de Newmark (1988, pg. 86), utilizado para descrever todo o tipo de mudança de categoria gramatical para resolver problemas de tradução pontuais.

O conceito relativamente recente das traduções naturais (natural translations) deveria ser incluído nesta listagem como termo relacionado com o conceito do bilinguismo na tradução, já com mais longa tradição nos estudos da tradução e que, por sua vez, está igualmente ausente desta proposta. Harris, foi, em 1973, um dos primeiros teóricos a estudar com alguma profundidade a relação entre o bilinguismo e a competência tradutiva, avançando com uma teoria da tradução natural que parte do princípio constitutivo de que existe uma relação coextensiva entre a competência bilingue e a capacidade de traduzir: "all bilinguals are able to translate, within the limits of their mastery of the two languages; therefore translating is coextensive with bilingualism" (Harris \& Sherwood 1978, pg. 155). Esta abordagem torna-se particularmente importante quando estuda a tradução 
natural efetuada por bilingues em situações espontâneas do quotidiano em comparação com a tradução profissional por tradutores com formação avançada em situações altamente estruturadas:

"Bilingualism and all forms of translation, whether the natural translation done «in everyday circumstances by bilinguals who have had no special training for it» (Harris 1976, pg. 96) or the professional translation of those with advanced translation degrees working in today's language industry, are necessarily connected at a very fundamental cognitive level." (Shreve 2012, pg. 1).

O facto de o bilinguismo na tradução ter sido, até este momento, um pouco negligenciado na Europa, ao contrário do que acontece nos EUA (cf. Antonini (2010)), pode explicar a ausência desta entrada na proposta de Vandepitte, mas, naturalmente, não a justifica, até porque se observa também na Europa um crescente interesse pelas redes de intérpretes e tradutores voluntários que, no curto, prazo irão modificar de forma significativa o panorama da tradução a nível global. À proposta de Vandepitte (2008, pgs. 584-585).

- Types by subject

NT: single-focus translation studies

NT: process-oriented translation studies

(incl. cognitive processes)

NT: studies of translation competence

$[\ldots]$

proponho, por estas razões, o seguinte acréscimo:

NT: studies of bilinguism in translation

RT: natural translation

RT: language brokering

RT: translation volunteers networks

NT: Babels

[2] ALARgamento DAS hiERARQUiAS ESTABELECIDAS POR VANDEPitTE

$O$ campo que se refere às metodologias contém um problema de hierarquização que resulta da relação de concomitância que existe, na minha opinião, entre os termos estudos da tradução indutivos (inductive translation studies), estudos da tradução baseados em corpora (corpus-based translation studies), abordagens hermenêuticas (hermeneutic approaches), estudos da tradução dedutivos (deductive translation studies), estudos da tradução experimentais (experimental translation studies) e abordagens especulativas (speculative approaches), por um lado, e abordagens qualitativas (qualitative approaches) e abordagens quantitativas (quantitative approaches), por outro, como se pode verificar na proposta da autora: 
- Types by method

Types by general research methods

NT: inductive translation studies

NT: corpus(-based) translation studies

NT: qualitative approaches

NT: quantitative approaches

NT: hermeneutic approaches

NT: deductive translation studies

NT: experimental translation studies

RT: think-aloud protocol studies

UF: TAP studies

NT: speculative approaches

(Vandepitte 2008, pg. 584)

Partilho com Flynn \& Gambier (2011, pg. 92-93) o pressuposto de que os métodos quantitativos e qualitativos, isoladamente ou em combinação, são aplicados a todos os trabalhos sobre os principais fatores do processo tradutivo:

"To recapitulate, two main methods of analysis can be used to study any of the four factors outlined above: quantitative or qualitative or a combination of the two. Listed under quantitative methods we have noted surveys, (cloze) tests, corpus analyses, key-logging, eye-tracking, screen-logging and related statistical analyses. Under qualitative methods we have noted various forms of text and discourse analysis, narrative and related studies, interviews with individuals or focus groups, think-aloud protocols, ethnographies, inquiries into to power, gender and other sets of relations."

Tendo em consideração este entendimento, impõe-se uma reorganização hierárquica dos tipos organizados por métodos de investigação geral da seguinte forma:

- Types by method

Types by general research methods

NT: inductive translation studies

NT: corpus(-based) translation studies

NT: qualitative approaches

NT: quantitative approaches

NT: hermeneutic approaches

NT: deductive translation studies

NT: experimental translation studies

RT: think-aloudprotocol studies

UF: TAP studies

NT: speculative approaches

(Vandepitte 2008, pg. 584)
- Types by method

Types by general research methods

NT: quantitative methods

NT: qualitative methods

RT: inductive approaches

RT: deductive approaches

RT: corpus(-based) approaches

NT: hermeneutic approaches

RT: experimental approaches

RT: think-aloud protocol studies

UF: TAP studies

NT: speculative approaches

(proposta do autor) 
A alteração de "inductive translation studies", "corpus(-bases) studies", "deductive translation studies" e "experimental translation studies" para "inductive approaches", "corpus(-based)approaches", "deductive approaches" e "experimental approaches" justifica-se, na minha opinião, por razões de coerência interna considerando que os itens em causa definem abordagens possíveis em que os métodos identificados podem vir a encontrar a sua aplicação.

\section{CONCLUS ÃO}

A construção de um thesaurus com estas características é uma tarefa complexa e exigente, e por natureza, discutível ou até polémica, porque as diferentes abordagens, práticas e objetivos nos estudos de tradução nem sempre encontram consenso sobre o que se pode considerar como nuclear e complementar (não no sentido da sua importância, mas sim da sua abrangência) mesmo dentro de cada ramo de investigação. Por essa razão, não proponho aqui uma alternativa de organização estrutural da área mas, pelo contrário, privilegio uma postura de cooperação construtiva na otimização de uma solução/proposta de cariz taxonómico.

No entanto, não quero deixar de mencionar a possibilidade de se pensar numa estruturação ontológica alternativa dos estudos de tradução. Flynn \& Gambier (2011), p. ex., consideram 4 fatores fundamentais e interligados na descrição e explicação da atividade tradutiva, a saber, 1 . discursos (no sentido lato incluindo traduções e toda a interação multilingual relacionada com o texto traduzido) 2 . práticas (para além da prática tradutiva, fatores múltiplos que de alguma forma a influenciam), 3. contextos (em que as traduções são produzidas) e 4. atores (que inclui todos aqueles que participam, direta ou indiretamente, na atividade tradutiva) (cf. Flynn \& Gambier (2011, pg.89-93). Na tentativa de assim agrupar os estudos pelo objetivo comum de procurar descobrir o que é ser tradutor (translatorship) talvez se poderia passar de uma perspetiva multidisciplinar que não procura necessariamente a integração do conhecimento, para um campo que Snell-Hornby et al. (1994) chamaram de uma disciplina interdisciplinar que, por sua vez, procura congregar todo o saber à volta daquilo que poderíamos chamar "translatorship". Uma ontologia, partindo deste pressuposto, teria desse modo, como fundamento estruturante alternativo os fatores que definem per se a tradução enquanto atividade humana comunicativa e intencional (cf. Vandepitte (2008, pg. 570). Porventura, seria um passo na direção, por muitos sentida como desejável, de uma reconciliação entre a teoria e a prática.

Agradeço à colega Anabela Barreiro a revisão atenta deste artigo. 


\section{REFERÊNCIAS}

Aitchison, Jean, Alan Gilchrist \& David Bawden. 2000. Thesaurus construction and use: A practical manual. Aslib IMI.

Antonini, Rachele. 2010. Natural translator and interpreter. Em Yves Gambier \& Luc van Doorslaer (eds.), Handbook of translation, vol. 2, 102-104. John Benjamins.

Baker, Mona (ed.). 1998. Routledge Encyclopedia of Translation Studies. Routledge.

Catford, John Cunnison. 1965. A linguistic theory of translation: An essay in applied linguistics. Oxford University Press.

Flynn, Peter \& Yves Gambier. 2011. Methodology in translation studies. Em Yves Gambier \& Luc van Doorslaer (eds.), Handbook of translation, vol. 2, 88-96. John Benjamins.

Gentzler, Edwin. 2001. Contemporary translation theories. Multilingual Matters 2nd edn.

Harris, Brian. 1976. The importance of natural translation. Working Papers in Bilingualism 12. 96-114.

Harris, Brian \& Bianca Sherwood. 1978. Translating as an innate skill. Em David Gerver \& H. Wallace Sinaiko (eds.), Language, interpretation and communication, 155-170. Plenum.

Hervey, Sándor \& Ian Higgins. 1992. Thinking translation. Routledge.

Holmes, James. 1987. The name and nature of translation studies. Em Gideon Toury (ed.), Translation across cultures, Bahri Publications.

Jääskeläinen, Riitta. 1993. Investigating translation strategies. Em John Laffling \& Sonja Tirkkonen-Condit (eds.), Recent Trends in Empirical Translation Research, 99-120. University of Joensuu.

Kuhiwczak, Piotr \& Karin Littau (eds.). 2007. A companion to translation studies. Multilingual Matters.

Munday, Jeremy (ed.). 2009. The Routledge Companion to Translation Studies. Routledge.

Newmark, Peter. 1988. Approaches to translation. Prentice Hall.

Popovic, Anton. 1976. Dictionary for the analysis of literary translation. Department of Comparative Literature, The University of Alberta. 
Pym, Anthony D. 2010. Exploring translation theories. Routledge.

Shreve, Gregory M. 2012. Bilingualism and translation. Em Yves Gambier \& Luc van Doorslaer (eds.), Handbook of translation, vol. 3, 1-6. John Benjamins.

Snell-Hornby, Mary, Franz Pöchhacker \& Klaus Kaindl (eds.). 1994. Translation Studies: An Interdiscipline. Selected papers from the Translation Studies Congress, Vienna, 1992. John Benjamins.

Tymoczko, Maria. 2005. Trajectories of research in translation studies. META 50(4). 1082-1097.

Vandepitte, Sonia. 2008. Remapping translation studies: towards a translation studies ontology. META 53(3). 569-588.

Venuti, Lawrence. 1998. Strategies of translation. Em Mana Baker (ed.), Encyclopedia of Translation Studies, 240-244. Routledge.

Williams, Jenny \& Andrew Chesterman. 2002. The map: A beginner's guide doing research in translation studies. St. Jerome Publishing.

CONTACTOS

Thomas J. C. Hüsgen

Faculdade de Letras da Universidade do Porto

thusgen@letras.up.pt 